\title{
Grand challenges in Milky Way and galaxies
}

\author{
Paola Marziani * \\ Osservatorio Astronomico di Padova, Istituto Nazionale di Astrofisica, Padova, Italy
}

Keywords: extragalactic astronomy, galaxies, Milky Way, active galactic nuclei, black holes, high redshift, quasars

\section{OPEN ACCESS}

\section{Edited and reviewed by:} Fabio La Franca,

Universita Roma Tre, Italy

*Correspondence:

Paola Marziani,

Osservatorio Astronomico di Padova, Istituto Nazionale di Astrofisica, Vicolo dell Osservatorio 5, 35122 Padova,

Italy

paola.marziani@oapd.inaf.it

Specialty section:

This article was submitted to Milky Way and Galaxies,

a section of the journal

Frontiers in Astronomy and Space

Sciences

Received: 29 April 2015 Accepted: 13 May 2015

Published: 27 May 2015

Citation:

Marziani P (2015) Grand challenges in Milky Way and galaxies.

Front. Astron. Space Sci. 2:1. doi: 10.3389/fspas.2015.00001
Extragalactic astronomy is a relatively young science. Its birth may be set at the time of the "great debate" between Harlow Shapley and Heber Curtis on the extragalactic nature of the nebulæ (which culminated with a meeting of the two protagonists in April 1920) or, with a more conservative stance, a few years later at the time of Edwin Hubble's discovery of Cepheid variables in Messier 31, the Andromeda galaxy and the nearest spiral galaxy to our own (Hubble, 1925). In the late 1920s the Kapteyns Universe (that posed the Sun as the center of the Milky Way) and Shapley's view (a giant Milky Way of which the spiral nebulæ were part) had fallen out of favor with the appreciation of the importance of interstellar matter in the absorption of light. Even if the Universe's constituents were stars and gas, they were not confined to a single galaxy, our own galaxy, the Milky Way. In the 1950s the recalibration of the relation between period and luminosity of the Cepheid stars provided a distance scale consistent with the one presently adopted, greatly increasing the distance of even the nearest spiral nebulæ and settling the issue of the island universes forever (Baade, 1958).

Frontiers in extragalactic astronomy - which, unlike other fields, can be literally associated with a physical distance-have progressed as improvements in instrumental capabilities made it possible to detect and to study more and more distant objects over an ever broader range of frequencies, from the radio to the $\gamma$-ray domain. Many results on distant sources are inferred by analogy with better studied sources, which are usually brighter and closer. Until now this approach-which is epistemologically risky (Salmon, 2012) - has not led research into major dead ends. There have been fully unexpected, and less unexpected discoveries that proved to be lasting paradigm shifts. The discovery of quasars expanded the cosmic scenario to distances previously unimaginable (Schmidt, 1963). The inference of dark matter in cluster of galaxies (Zwicky, 1933), and of dark matter influence on the rotation curve of galaxies (Rubin et al., 1980) in the early 1970s provided early glimpses of the "dark Universe" as we understand it today. The realization of the importance of obscuration and extinction phenomena that affect even the most powerful extragalactic sources came gradually with the development of X-ray, spectro-polarimetric and IR instrumentation. Other discoveries fulfilled dreams that had become reality, like the first planetary transit in front of a Milky Way star that was not the Sun (Charbonneau et al., 2000). All of them enriched our view of the Universe. For comprehensive reviews, the reader may consider the historical chapters of D'Onofrio et al. (2012) and D'Onofrio et al. (2015) that provide first-hand accounts on major discoveries of the last 50 years of Galactic and extra-galactic astronomical research. The possibility of a truly revolutionary paradigm change was vented perhaps only once, at the time of quasars discovery (Arp, 1987). In the mid 1960s quasar distances derived from the Hubble law looked enormous, and the ensuing quasar power demanded physics that appeared exotic at the time: accretion onto a supermassive black hole (Salpeter, 1964; Zel'Dovich and Novikov, 1965). The issue was definitely settled when astronomers could see better at larger distances. With the advent of Hubble Space Telescope in the early 1990s quasars were definitely confirmed as nuclei of distant galaxies as implied by Hubble's law (Bahcall et al., 1997). Active galactic nuclei (AGN) — which include quasars that were once believed to be rare-were found to be relatively common and to play an important role in the evolution of galaxies (e.g., Kormendy and Ho, 2013, and references therein). 
These considerations lead to the framing of three challenges in Galactic and extra-galactic astronomy that, in my opinion, can be legitimately called "grand:" the expansion of the redshift frontier, the census of galaxies and of their constituents with a full understanding of galactic evolution, and the study of accretion onto supermassive black holes in galactic nuclei, whose event horizon is literally a physical frontier of the Universe and where there is a potential for the development of new physics.

The first grand challenge is obviously associated with data improvement in terms of sample coverage and multiplexing for the most distant galaxies. There has always been a redshift frontier in extragalactic astronomy, and expanding the present one at $z \approx 10$ (Ono et al., 2013) is perhaps the ultimate challenge since we are dealing with cosmic epoch when the universe was going opaque to UV radiation. Where does the re-ionization energy come from, from star forming galaxies or massive black holes (Madau et al., 1999)? Primordial galaxies did not live through the evolutionary paths of present-day galaxies so that they provide the best representation of galaxies not so much time after formation. However, it is still unknown at which epoch after the Big Bag massive progenitor could exist. There is a cosmic age problem, as several evolved galaxies have been found a relatively high- $z$ (e.g., Riechers et al., 2013; Watson et al., 2015). A special population of-highly accreting quasars has to be assumed to explain the large masses observed at high $z$ (Netzer and Trakhtenbrot, 2014). At $z \approx 6$ we still observe supersolar iron abundances which may conflict with timescales needed for iron enrichment due to type Ia supernovæ (Kurk et al., 2007). The shifting redshift frontier, that 30 years ago was at $z \approx 3.5$ (Trimble and Woltjer, 1986) has always yielded implications on galaxy formation and evolution; far-reaching implication may soon even involve $\Lambda$-Cold Dark Matter cosmology. IR and sub$\mathrm{mm}$ instrumentation from ground and space (such as the James Webb Space Telescope, optimized for IR) is and will be at the forefront of the observation of the most distant galaxies and quasars.

The second grand challenge is the development of a census of sources-let it be the stars in the Milky Way or galaxies at remote cosmic epochs - that may lead to a full understanding of the Milky Way and external galaxies structure and evolution. We live in an exciting time when the census of the stellar populations within the Galaxy and of the extra-galactic Universe is still being expanded to an unprecedented extent of completeness through surveys such as the new generation of the Sloan Digitized Sky Survey (SDSS) that provide immense public databases. Dedicated surveys are being carried out to map the detailed threedimensional structure of the Galaxy: the space-based Gaia as well as spin-offs of the Sloan IR surveys that overcome the effect of interstellar extinction especially heavy toward the direction of the Galactic center. Planned wide-field telescopes will provide an almost synoptic survey of the whole sky at an unprecedented rate (Ivezic et al., 2008).

We still miss a large population of quiescent and active galaxies that are too faint for the present day instrumentation, and not just the ones at the highest $z$ that are being studied by ultra-deep Hubble Space Telescope observations. Recent studies based on the SDSS still miss the faint companions and important morphological features (Smirnova et al., 2010). Even in the Local Group, the census of all dwarf galaxies may not be complete (Makarov et al., 2012). This is an unfortunate circumstance since dwarf galaxies are directly related to galaxy formation theories, and minor mergers are expected to play an important role in the evolution of bulges (Naab et al., 2009). There is an obvious bias in AGN discovery that depends on black hole mass and accretion rate in flux-limited samples, so that at high $z$ we still miss a large population of quasars (Sulentic et al., 2014).

Galaxy evolution involves a number of non-linear processes in structure formation that are still poorly understood. Environmental effects such as gravitational interaction between galaxies are a fundamental driver of galactic evolution though hierarchical growth (Hopkins et al., 2006), and induced star formation (e.g., Mihos and Hernquist, 1996; Krongold et al., 2002). Environmental effects on galaxies can be as strong as to lead to a morphological transformation through merging (Toomre and Toomre, 1972), and through the depletion and stripping of the galaxy interstellar medium (Spitzer and Baade, 1951). They yield evolutionary patterns that can be radically different from galaxy to galaxy. Only in recent years environmental phenomena have been studied in a detailed way that makes it possible to appreciate their complexity (e.g., Förster Schreiber et al., 2009; Monreal-Ibero et al., 2010; Sivanandam et al., 2014, for different approaches), although we still miss many aspects of the star formation micro-physics (Evans, 1999) and multifrequency observational data are often presented on an object-by-object basis. Realistic models of galactic structure and evolution should include evolving stellar populations, the supermassive black hole, and their feedback on the cold and hot components of the inter-stellar medium (Fabian, 2012) in a largescale environmental context. We still need a full appreciation of the relative importance of primordial collapse, hierarchical growth, secular evolution and active nucleus feedback to account for empirical relations such as the fundamental plane of spheroidal galaxies (Djorgovski and Davis, 1987; D’Onofrio et al., 2013), that implies different stellar population for different galactic structures, or color-magnitude diagrams, where the two fundamental sequences of galaxies, the blue (star-forming) and red (quiescent) one are defined.

The third grand challenge is to assess the role of accretion onto supermassive compact objects in galactic structure and evolution. Unification schemes account for a majority population of obscured quasars and a small minority of beamed sources (Antonucci, 1993; Urry and Padovani, 1995), but do not provide a scenario for the diversity of unobscured, unbeamed AGNs. Is the so-called first eigenvector of quasars (Boroson and Green, 1992; Sulentic et al., 2000) a real surrogate of the stellar HertzsprungRussell diagram? There is a well-defined sequence that allows for the definition of spectral types. The sequence is likely to be governed by Eddington ratio, but orientation, mass, spin, luminosity, chemical composition and other effects complicate its interpretation (e.g., Marziani et al., 2001; Shen and Ho, 2014; Wang et al., 2014). Accretion processes may be almost selfsimilar over a huge range of masses (Zamanov and Marziani, 2002; Merloni et al., 2003) but they are still poorly understood as far as the structure of the accretion flow is concerned (e.g., 
Abramowicz and Straub, 2014, and references therein), especially if the flow is advection-dominated at very low and high accretion rates. How and under which condition will they launch a jet or a radiation driven wind? There is no clear physical understanding of why AGN come into two varieties, radio quiet and radio loud. Hierarchical growth scenarios also predict a large number of subparsec binary black holes (Volonteri et al., 2009), but where are they? Until now their existence is supported by observations only in a handful of cases (Bon et al., 2012). As in other fields of extragalactic research, there is also here an obvious gap between theory and observation.

There is convincing evidence of a large concentration of mass in at least some galactic nuclei (Peterson and Wandel, 1999; Ghez et al., 2003). Is the mass really in the form of a black hole? An important aspect of the third challenge is the ability to resolve the inner structure of galactic nuclei down to the black hole event horizon, not only because of its uncertain nature and the possibility of new physics (Hawking, 2014), but also because within a few tens of gravitational radii from the event horizon it is possible to test general relativity, the physics of accretion, as well as the collimation of relativistic jets. In principle, with enough resolving power in the sub$\mathrm{mm}$ domain it is possible to measure the black hole spin and even detect possible deviations from the Kerr metric i.e.,

\section{References}

Abramowicz, M. A., and Straub, O. (2014). Accretion discs. Scholarpedia 9:2408. doi: $10.4249 /$ scholarpedia. 2408

Antonucci, R. (1993). Unified models for active galactic nuclei and quasars. Ann. Rev. Astron. Astrophys. 31, 473-521. doi: 10.1146/annurev.aa.31.090193. 002353

Arp, H. (1987). Quasars, Redshifts, and Controversies. Berkeley: Interstellar Media. Baade, W. (1958). Problems in the determination of the distance of galaxies. Astron. J. 63, 207-210. doi: 10.1086/107726

Bahcall, J. N., Kirhakos, S., Saxe, D. H., and Schneider, D. P. (1997). Hubble space telescope images of a sample of 20 nearby luminous quasars. Astrophys. J. 479, 642-658. doi: 10.1086/303926

Bon E., Jovanović, P., Marziani, P., Shapovalova, A. I., Bon, N., Borka Jovanović, V., et al. (2012). The first spectroscopically resolved sub-parsec orbit of a supermassive binary black hole. Astrophys. J. 759:118. doi: 10.1088/0004$637 \mathrm{X} / 759 / 2 / 118$

Boroson, T. A., and Green, R. F. (1992). The emission-line properties of low-redshift quasi-stellar objects. Astrophys. J. Suppl. Ser. 80, 109-135. doi: $10.1086 / 191661$

Broderick, A. E., Johannsen, T., Loeb, A., and Psaltis, D. (2014). Testing the no-hair theorem with event horizon telescope observations of sagittarius $\mathrm{A}^{*}$. Astrophys. J. 784:7. doi: 10.1088/0004-637X/784/1/7

Charbonneau, D., Brown, T. M., Latham, D. W., and Mayor, M. (2000). Detection of planetary transits across a sun-like star. Astrophys. J. Lett. 529, L45-L48. doi: $10.1086 / 312457$

Djorgovski, S., and Davis, M. (1987). Fundamental properties of elliptical galaxies. Astrophys. J. 313, 59-68. doi: 10.1086/164948

D'Onofrio, M., Marziani P., and Sulentic, J. W., (eds.). (2012). Fifty Years of Quasars From Early Observations and Ideas to Future Research, Astrophysics and Space Science Library, Vol. 386. Berlin-Heidelberg: Springer Verlag.

D’Onofrio, M., Fasano, G., Moretti, A., Marziani, P., Bindoni, D., Fritz, J., et al. (2013). The hybrid solution for the fundamental plane. Mon. Not. R. Astron. Soc. 435, 45-63. doi: 10.1093/mnras/stt1278 to even test the so-called "no hair" theorem for black holes (Broderick et al., 2014). This is the challenge meant for the Event Horizon Telescope (EHT), a very long baseline interferometry sub-milimetre array that is presently under development (Ricarte and Dexter, 2015), and for the planned Athena X-ray telescope.

The time is ripe to work in the direction of a coherent view in galaxy evolution that will include accretion processes in the framework of a less and less lacunose mapping of the Galactic and of the distant Universe. Facing these challenges calls for improved light gathering power that is needed for the high $\mathrm{S} / \mathrm{N}$ and high-resolution spectroscopy of faint sources-especially in the IR and X-ray bands, to observe dust-obscured or highredshift sources-that will be detected in a large numbers by the wide field telescopes expected to come into operation in the next years. It will require the dedication and the enthusiasm of the present and next generations of researchers.

\section{Acknowledgments}

I would like to acknowledge Deborah Dultzin and Jack Sulentic for many years of encouragement and support. I also thank Mauro D'Onofrio and Sam Finn for a careful reading of the manuscript.
D’Onofrio, M., Rampazzo, R., and Zaggia, S., (eds.). (2015). From the Realm of Nebulae to the Society of Galaxies, Astrophysics and Space Science Library. Berlin-Heidelberg: Springer Verlag.

Evans, N. J. II. (1999). Physical conditions in regions of star formation. Ann. Rev. Astron. Astrophys. 37, 311-362. doi: 10.1146/annurev.astro.37.1.311

Förster Schreiber, N. M., Genzel, R., Bouché, N., Cresci, G., Davies, R., Buschkamp, P., et al. (2009). The SINS survey: SINFONI integral field spectroscopy of z 2 star-forming galaxies. Astrophys. J. 706, 1364-1428. doi: 10.1088/0004$637 \mathrm{X} / 706 / 2 / 1364$

Fabian, A. C. (2012). Observational evidence of active galactic nuclei feedback. Ann. Rev. Astron. Astrophys. 50, 455-489. doi: 10.1146/annurev-astro-081811125521

Ghez, A. M., Duchêne, G., Matthews, K., Hornstein, S. D., Tanner, A., Larkin, J., et al. (2003). The first measurement of spectral Lines in a short-period star bound to the galaxy's central black hole: a paradox of youth. Astrophys. J. Lett. 586, L127-L131. doi: 10.1086/374804

Hawking, S. W. (2014). Information preservation and weather forecasting for black holes. arXiv: 1401.5761

Hopkins, P. F., Hernquist, L., Cox, T. J., Di Matteo, T., Robertson, B., and Springel, V. (2006). A unified, merger-driven model of the origin of starbursts, quasars, the cosmic x-ray background, supermassive black holes, and galaxy spheroids. Astrophys. J. Suppl. 163, 1-49. doi: 10.1086/499298

Hubble, E. P. (1925). Cepheids in spiral nebulae. Observatory 48, 139-142.

Ivezic, Z., Tyson, J. A., Abel, B., Acosta, E., Allsman, R., AlSayyad, Y., et al. (2008). LSST: from science drivers to reference design and anticipated data products. arXiv: 0805.2366v4

Kormendy, J., and Ho, L. C. (2013). Coevolution (or not) of supermassive black holes and host galaxies. Ann. Rev. Astron. Astrophys. 51, 511-653. doi: 10.1146/annurev-astro-082708-101811

Krongold, Y., Dultzin-Hacyan, D., and Marziani, P. (2002). The circumgalactic environment of bright IRAS galaxies. Astrophys. J. 572, 169-177. doi: $10.1086 / 340299$

Kurk, J. D., Walter, F., Fan, X., Jiang, L., Riechers, D. A., Rix, H. W., et al. (2007) Black hole masses and enrichment of z 6 SDSS quasars. Astrophys. J. 669, 32-44. doi: $10.1086 / 521596$ 
Madau, P., Haardt, F., and Rees, M. J. (1999). Radiative transfer in a clumpy universe. III. The nature of cosmological ionizing sources. Astrophys. J. 514, 648-659. doi: 10.1086/306975

Makarov, D., Makarova, L., Sharina, M., Uklein, R., Tikhonov, A., Guhathakurta, P., et al. (2012). A unique isolated dwarf spheroidal galaxy at $\mathrm{D}=1.9 \mathrm{Mpc}$. Mon. Not. R. Astron. Soc. 425, 709-719. doi: 10.1111/j.1365-2966.2012.21581.x

Marziani, P., Sulentic, J. W., Zwitter, T., Dultzin-Hacyan, D., and Calvani, M. (2001). Searching for the physical drivers of the eigenvector 1 correlation space. Astrophys. J. 558, 553-560. doi: 10.1086/322286

Merloni, A., Heinz, S., and di Matteo, T. (2003). A fundamental plane of black hole activity. Mon. Not. R. Astron. Soc. 345, 1057-1076. doi: 10.1046/j.13652966.2003.07017.x

Mihos, J. C., and Hernquist, L. (1996). Gasdynamics and starbursts in major mergers. Astrophys. J. 464, 641. doi: 10.1086/177353

Monreal-Ibero, A., Arribas, S., Colina, L., Rodríguez-Zaurín, J., Alonso-Herrero, A., and García-Marín, M. (2010). VLT-VIMOS integral field spectroscopy of luminous and ultraluminous infrared galaxies. II. Evidence for shock ionization caused by tidal forces in the extra-nuclear regions of interacting and merging LIRGs. Astron. Astrophys. 517, A28. doi: 10.1051/0004-6361/200913239

Naab, T., Johansson, P. H., and Ostriker, J. P. (2009). Minor mergers and the size evolution of elliptical galaxies. Astroph. J. Lett. 699, L178-L182. doi: 10.1088/0004-637X/699/2/L178

Netzer, H., and Trakhtenbrot, B. (2014). Bolometric luminosity black hole growth time and slim accretion discs in active galactic nuclei. Mon. Not. R. Astron. Soc. 438, 672-679. doi: 10.1093/mnras/stt2238

Ono, Y., Ouchi, M., Curtis-Lake, E., Schenker, M. A., Ellis, R. S., McLure, R. J., et al. (2013). Evolution of the sizes of galaxies over $7<\mathrm{z}<12$ revealed by the 2012 hubble ultra deep field campaign. Astrophys. J. 777:155. doi: 10.1088/0004-637X/777/2/155

Peterson, B. M., and Wandel, A. (1999). Keplerian motion of broad-line region gas as evidence for supermassive black holes in active galactic nuclei. Astrophys. J. Lett. 521, L95-L98. doi: 10.1086/312190

Ricarte, A., and Dexter, J. (2015). The event horizon telescope: exploring strong gravity and accretion physics. Mon. Not. R. Astron. Soc. 446, 1973-1987. doi: 10.1093/mnras/stu2128

Riechers, D. A., Bradford, C. M., Clements, D. L., Dowell, C. D., Pérez-Fournon, I., Ivison, R. J., et al. (2013). A dust-obscured massive maximum-starburst galaxy at a redshift of 6.34. Nature 496, 329-333. doi: 10.1038/nature12050

Rubin, V. C., Ford, W. K. J., and Thonnard, N. (1980). Rotational properties of 21 SC galaxies with a large range of luminosities and radii, from NGC $4605 / \mathrm{R}=4 \mathrm{kpc} /$ to UGC $2885 / \mathrm{R}=122 \mathrm{kpc} /$. Astrophys. J. 238, 471-487. doi: $10.1086 / 158003$

Salmon, M. (2012). Introduction to Logic and Critical Thinking. Boston, MA: Cengage Learning.

Salpeter, E. E. (1964). Accretion of interstellar matter by massive objects. Astrophys. J. 140, 796-800.

Schmidt, M. (1963). 3C 273 : a star-like object with large red-shift. Nature 197:1040. doi: $10.1038 / 1971040 \mathrm{a} 0$

Shen, Y., and Ho, L. C. (2014). The diversity of quasars unified by accretion and orientation. Nature 513, 210-213. doi: 10.1038/nature13712
Sivanandam, S., Rieke, M. J., and Rieke, G. H. (2014). Tracing ram-pressure stripping with warm molecular hydrogen emission. Astrophys. J. 796:89. doi: 10.1088/0004-637X/796/2/89

Smirnova, A. A., Moiseev, A. V., and Afanasiev, V. L. (2010). Seyfert galaxies that are undergoing merging but appear non-interacting. Mon. Not. R. Astron. Soc. 408, 400-406. doi: 10.1111/j.1365-2966.2010. 17121.x

Spitzer, L. Jr., and Baade, W. (1951). Stellar populations and collisions of galaxies. Astrophys. J. 113, 413. doi: 10.1086/145406

Sulentic, J. W., Zwitter, T., Marziani, P., and Dultzin-Hacyan, D. (2000). Eigenvector 1: an optimal correlation space for active galactic nuclei. Astrophys. J. Lett. 536, L5-L9. doi: 10.1086/312717

Sulentic, J. W., Marziani, P., del Olmo, A., Dultzin, D., Perea, J., and Alenka Negrete, C. (2014). GTC spectra of $\mathrm{z} \approx 2.3$ quasars: comparison with local luminosity analogs. Asttron. Astrophys. 570, A96. doi: 10.1051/0004$6361 / 201423975$

Toomre, A., and Toomre, J. (1972). Galactic bridges and tails. Astrophys. J. 178, 623-666.

Trimble, V., and Woltjer, L. (1986). Quasars at 25. Science 234, 155-161. doi: $10.1086 / 151823$

Urry, C. M., and Padovani, P. (1995). Unified schemes for radio-loud active galactic nuclei. Publ. Astron. Soc. Pac. 107, 803. doi: 10.1086/133630

Volonteri, M., Miller, J. M., and Dotti, M. (2009). Sub-parsec supermassive binary quasars: expectations at $\mathrm{z}<1$. Astrophys. J. Lett. 703, L86-L89. doi: 10.1088/0004-637X/703/1/L86

Wang, J. M., Du, P., Li, Y. R., Ho, L. C., Hu, C., and Bai, J. M. (2014). A new approach to constrain black hole spins in active galaxies using optical reverberation mapping. Astrophys. J. Lett. 792:L13. doi: 10.1088/20418205/792/1/L13

Watson, D., Christensen, L., Knudsen, K. K., Richard, J., Gallazzi, A., and Michałowski, M. J. (2015). A dusty, normal galaxy in the epoch of reionization. Nature 519, 327-330. doi: 10.1038/nature14164

Zamanov, R., and Marziani, P. (2002). Searching for the physical drivers of eigenvector 1: from quasars to nanoquasars. Astrophys. J. Lett. 571, L77-L80. doi: $10.1086 / 341367$

Zel’Dovich, Y. B., and Novikov, I. D. (1965). Mass of quasi-stellar objects. Sov. Phys Dokl. 9, 834 .

Zwicky, F. (1933). Die rotverschiebung von extragalaktischen nebeln. Helv. Phys Acta 6, 110-127.

Conflict of Interest Statement: The author declares that the research was conducted in the absence of any commercial or financial relationships that could be construed as a potential conflict of interest.

Copyright (c) 2015 Marziani. This is an open-access article distributed under the terms of the Creative Commons Attribution License (CC BY). The use, distribution or reproduction in other forums is permitted, provided the original author(s) or licensor are credited and that the original publication in this journal is cited, in accordance with accepted academic practice. No use, distribution or reproduction is permitted which does not comply with these terms 